\title{
Determination of the Heave and Pitch Motions of a Floating Cylinder by Analytical Solution of its Diffraction Problem and Examination of the Effects of Geometric Parameters on its Dynamics in Regular Waves
}

\author{
Parviz Ghadimi, Hadi Paselar Bandari, Ali Bakhshandeh Rostami \\ Department of Marine Technology, Amirkabir University of Technology, Tehran, Iran \\ E-mail: pghadimi@aut.ac.ir, hpaselar@aut.ac.ir, rostami115@yahoo.com
}

\begin{abstract}
In this paper, diffraction problem of a floating vertical circular cylinder is studied. Detailed analytical solution for the boundary value problem to evaluate the wave loads for the cylinder with heave and pitch motions in water of finite depth in the presence of an incident wave, has been presented. Accordingly, diffraction problem for a truncated vertical circular cylinder of radius $a$ in water of finite depth $d$ has been considered. Incident wave used in this problem, is assumed to be linear with amplitude $H$. In order to calculate the added mass and damping coefficients of the pitch motion of the body, strip theory is applied. For the heave motion, the hydrodynamic coefficients are found by the submerged disk method. Parametric studies of the effects of the wave excitation force on the floating cylinder have been performed for two different cases: 1) constant draft of the cylinder with varying water depth and 2) varying draft of the cylinder with constant water depth.
\end{abstract}

Keywords: Diffraction Phenomenon; Floating Circular Cylinder; Dynamic Motion; Strip Theory; Submerged Disk Method

\section{Introduction}

It is well known that floating structures such as ocean platforms, breakwaters, and wave energy devices are often used in ocean engineering. The hydrodynamic properties, of which most important are the hydrodynamic coefficients, wave 
excitation forces, transmission coefficient, and reflection coefficient, are of major interest for designers and many researches have been carried out and several results have been obtained.

Accurate prediction of the wave loads exerted by surface waves on rigid structures is imperative for designing the offshore structures. To analyze the hydrodynamic properties of floating structures, various methods such as Boundary Element Method (BEM), Finite Element Method (FEM), and some analytical methods can be used [1]. Among all the existing methods, the most efficient is analytical method, which is only applicable to some particular problems. For example, vertical circular cylinders are among the best marine elements that are utilized in the construction of many offshore platforms such as Spars, Semisubmersible, TLPs, etc. Numerical methods like BEM and FEM are suitable for general problems, but the computational procedure is complex, while the efficiency and accuracy of the results are relatively lower than those of the analytical methods. Therefore, the most suitable methods for some particular problems still remain to be the analytical methods [1].

Up to now, many scholars have applied various analytical methods. Among them are Miles and Gilbert [2] who studied the diffraction problem of surface waves on a circular dock and subsequently Garret [3] presented the results for the horizontal and vertical forces and moments on the dock. Black, Mei and Bray [4] calculated the wave forces on a truncated cylinder that either extended to the free surface or rested on the seabed. Isaacson [5] extended Garrett's method to a submerged truncated cylinder sitting on the seabed. Yeung [6], Sabuncu and Calisal [7,8,9] investigated a set of theoretical added mass and damping coefficients for a floating circular cylinder in water of finite depth. Yeung and Sphaier [10] determined the radiation and diffraction properties of a floating vertical cylinder of finite draft in a channel. Berggren and Johansson [11] and Eidsmoen [12] presented the heave radiation problem of a two-body axisymmetric system and calculated the heave added masses and damping coefficients. Bhatta and Rahman $[13,14]$ calculated the analytical and numerical results for diffraction loads and radiation coefficients for a vertical circular cylinder in a finite water depth. Williams et al. [15] studied the radiation and diffraction by a single floating circular cylinder and obtained theoretical results of hydrodynamic coefficients and the wave forces. Wu et al. [16] explored the diffraction and radiation problem for a cylinder over a caisson in water of finite depth and presented the calculated results of hydrodynamic coefficients, wave forces and hydrodynamic effect of the caisson on the hydrodynamics of the cylinder. In order to achieve findings close to the experiment results, a second-order diffraction problem for truncated cylinder in finite depth water was investigated by Huang and Eatock Taylor [17] and Mavrakos and Chatjigeorgiou [18] and diffraction forces due to second-order potential was calculated.

In this paper, we have considered the diffraction problem of a floating vertical circular cylinder. Accordingly, we have presented the analytical solution for the boundary value problem to evaluate the wave loads for a vertical circular cylinder 
with heave and pitch motion in water of finite depth in the presence of an incident wave. To accomplish this, strip theory has been applied for determination of the damping coefficients of the pitch motion of the body, while the submerged disk method has been used for calculation of the hydrodynamic coefficients of the heave motion of the body.

\section{Mathematical Formulation}

In this article, effort has been made to investigate the effects of geometrical parameters on the hydrodynamics of a floating cylinder. Diffraction problem for a truncated vertical circular cylinder of radius $a$ in water of finite depth $d$ has been considered. Incident wave utilized in this problem is linear with amplitude $H$. The geometry and wave characteristics are shown in Fig.1. The $z$-axis in the Cartesian coordinate system $O x y z$ is vertical upward from the seabed, $x$-axis in direction of wave motion and $y$-axis is perpendicular to it. However, in the cylindrical coordinate system $\operatorname{Or} \theta z, r$-axis is in the direction of the cylinder radius and $\theta$-axis is in the counterclockwise angular direction from the positive $x$ axis.

This is a floating cylinder without any mooring system which is freely moving, but its motion in direction of wave propagation (i.e. surge) is ignored. Also, in this problem it is assumed that the fluid is incompressible, inviscid and the fluid motion is irrotational.

The fluid region under the water surface is divided into two general regions; an internal region of radius $r \leq a$ and depth of $0 \leq z \leq h$ under the cylinder and an external region of radius $r \geq a$ extending from the seabed to the water surface (i.e. $0 \leq z \leq d$ ) around the cylinder. Furthermore, the external region of $r=a$ is split into two sub-regions: 1) from the seabed to the height $h$ where the continuity condition is applied, and 2) from the height $h$ to the water free surface over which the impenetrability condition is applied.

\subsection{Governing Equations and Boundary Conditions}

In the present paper, a vertical circular cylinder of radius $a$ is assumed to be located in an incompressible, inviscid, and irrotational fluid with a finite velocity. Based on the stated conditions, the governing equation for the flow field is the Laplace's equation. For solving the Laplaces' equation, it is necessary to identify the physical boundary condition. One of these required conditions is the linear free surface boundary condition which is given in Eq.2.

Governing equation with boundary conditions are represented here as

$$
\begin{aligned}
& \nabla^{2} \varphi=0 \\
& \frac{\partial^{2} \varphi}{\partial t^{2}}+g \frac{\partial \varphi}{\partial z}=0 \quad \text { on } \quad z=d
\end{aligned}
$$


In equations 3 to 5 , the impenetrability condition on the seabed and on the body surface, are presented as

$\begin{array}{lll}\frac{\partial \varphi}{\partial z}=0 & \text { on } & z=0 \\ \frac{\partial \varphi}{\partial z}=0 & \text { on } & z=h \\ \frac{\partial \varphi}{\partial r}=0 & \text { on } & r=a\end{array}$

Ultimately, the far-field boundary condition (i.e. Sommerfeld radiation condition) is written in Eq.6. as

$\lim _{r \rightarrow \infty} \sqrt{r}\left(\frac{\partial \varphi_{s}}{\partial r}-i k_{0} \varphi_{s}\right)=0$.

One of the important characteristics of the Laplace's equation is its linear characteristic. This property makes it a perfect candidate for applying the principle of superposition in order to find its solution. Potentials for regions 1 and 2 are denoted by $\varphi_{1}$ and $\varphi_{2}$, respectively. Therefore, the total potential, based on the principle of superposition, is the sum of these potentials as in

$\varphi=\varphi_{1}+\varphi_{2}$.

The problem separates into an interior domain (beneath the cylinder) and an exterior domain. Furthermore, the latter is a diffraction problem and consists of incident and scattered potentials. Also, boundary condition (Eq.6) is satisfied by the scattered potential $\varphi_{s}$ and is known as the Sommerfeld radiation condition, where $k_{0}$ is the wave number.

$\varphi_{2}=\varphi_{I 2}+\varphi_{s 2}$.

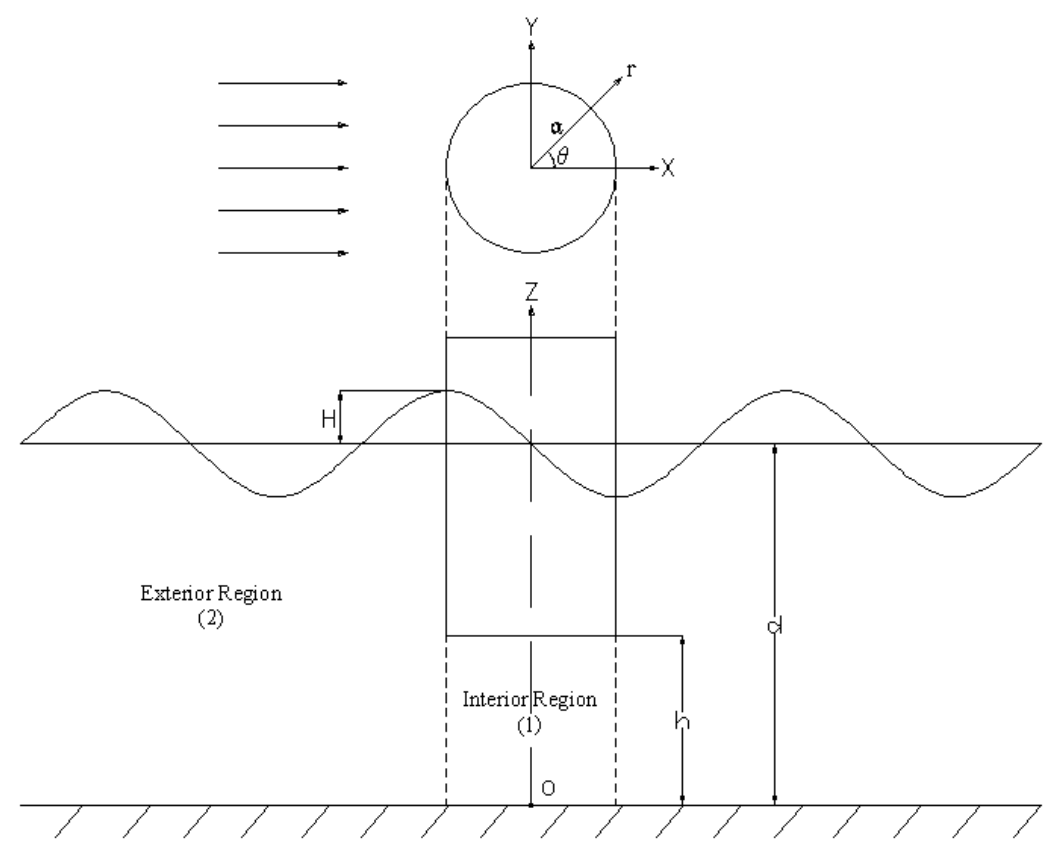

Fig.1. Domain geometry. 


\section{Solutions}

\subsection{Interior and Exterior Solutions}

For the incompressible, inviscid and irrotational fluid, the velocity potential $\emptyset(r, \theta, z, t)$ can be introduced as $\emptyset(r, \theta, z, t)=\operatorname{Re}\left[\varphi(r, \theta, z) e^{-i \omega t}\right]$ which is the real part of complex expression. $\varphi$ is the special potential and $\omega$ is the angular frequency of the wave.

- Potential in the interior region

Governing equation for the potential in the interior region is as follows:

$$
\begin{aligned}
& \nabla^{2} \varphi_{1}=0 \\
& \frac{\partial \varphi_{1}}{\partial z}=0 \quad \text { on } \quad z=0 \\
& \frac{\partial \varphi_{1}}{\partial z}=0 \quad \text { on } \quad z=h
\end{aligned}
$$

Using the method of separation of variables, the procedure of solving problem is performed as follows:

$\varphi_{1}(r, \theta, z)=R(r) \cdot \Theta(\theta) \cdot Z(z)$

Substituting (12) in Laplace's equation (Eq.9):

$$
\frac{r^{2} R^{\prime \prime}+r R^{\prime}}{R}+r^{2} \frac{Z^{\prime \prime}}{Z}=-\frac{\Theta^{\prime \prime}}{\Theta}=\lambda, \quad \frac{Z^{\prime \prime}}{Z}=-\gamma
$$

Both sides of Eq.13 must equal the same constant $\lambda$ whereas $\frac{Z^{\prime \prime}}{Z}$ is assumed to be the arbitrary constant $\gamma$. Equation (13) yields in three ordinary differential equations:

$\frac{d^{2} \Theta}{d \theta^{2}}+\lambda \Theta=0 \quad, \quad \frac{d^{2} Z}{d z^{2}}+\gamma Z=0 \quad, \quad r^{2} \frac{d^{2} R}{d r^{2}}+r \frac{d R}{d r}-\left(\gamma r^{2}+\lambda\right) R=0$

Thus, the potential in the interior region that satisfies (9), (10) and (11) is given as follows:

$$
\begin{aligned}
& \emptyset_{1}(r, \theta, z, t)=\sum_{n=-\infty}^{\infty} \chi_{n}^{1}(r, z) e^{i n \theta} e^{-i \omega t} \\
& \chi_{n}^{1}(r, z)=\frac{C_{n 0}}{2}\left(\frac{r}{a}\right)^{|n|}+\sum_{m=1}^{\infty} C_{n m} \frac{I_{n}\left(\frac{m \pi r}{h}\right)}{I_{n}\left(\frac{m \pi a}{h}\right)} \cos \left(\frac{m \pi z}{h}\right)
\end{aligned}
$$


where $I_{n}\left(\frac{m \pi r}{h}\right)$ is the modified Bessel function of first kind and order $n$. The Fourier coefficient $C_{n m}$ is given by:

$C_{n m}=\frac{2}{h} \int_{0}^{h} \chi_{n}^{1}(a, z) \cos \left(\frac{m \pi z}{h}\right) d z$

\section{- $\quad$ Potential in the exterior region}

Governing equation and boundary conditions to obtain the velocity potential in the exterior domain are listed as

$$
\begin{aligned}
& \nabla^{2} \varphi_{2}=0 \\
& \frac{\partial^{2} \varphi_{2}}{\partial t^{2}}+g \frac{\partial \varphi_{2}}{\partial z}=0 \quad \text { on } \quad z=d \\
& \frac{\partial \varphi_{2}}{\partial z}=0 \quad \text { on } z=0 \\
& \frac{\partial \varphi_{2}}{\partial r}=0 \text { on } r=a \\
& \lim _{r \rightarrow \infty} \sqrt{r}\left(\frac{\partial \varphi_{2 s}}{\partial r}-i k_{0} \varphi_{2 s}\right)=0
\end{aligned}
$$

The incident wave potential satisfying Eq.18 and boundary conditions (19) and (20) is determined to be

$\varphi_{2 I}=-\frac{g H}{\omega} \frac{\cosh \left(k_{0} z\right)}{\cosh \left(k_{0} d\right)} \sum_{n=-\infty}^{\infty} i^{n} J_{n}\left(k_{0} r\right) e^{i n \theta} e^{-i \omega t}$

where $J_{n}\left(k_{0} r\right)$ is the Bessel function of first kind and order $n$.

The scattered wave potential in the exterior region must satisfy (18), (19), (20), (21) and (22). Therefore using the separation of variables method, as before, we have:

$\varphi_{2 s}(r, \theta, z)=R(r) \cdot \Theta(\theta) \cdot Z(z)$

Substituting (24) in Laplace's equation (18), yields (25):

$\frac{d^{2} \Theta}{d \theta^{2}}+\lambda \Theta=0 \quad, \quad \frac{d^{2} Z}{d z^{2}}+\gamma Z=0 \quad, \quad r^{2} \frac{d^{2} R}{d r^{2}}+r \frac{d R}{d r}-\left(\gamma r^{2}+\lambda\right) R=0$

Hence, one can easily derive the expressions for the scattered potential as $\varphi_{2 s}(r, \theta, z)=$ $\sum_{n=-\infty}^{\infty}\left\{\cosh \left(k_{0} z\right)\left[C_{5} J_{n}\left(k_{0} r\right)+C_{6} Y_{n}\left(k_{0} r\right)\right]+\sum_{p=1}^{\infty} \cos \left(k_{p} z\right)\left[C_{7} I_{n}\left(k_{p} r\right)+\right.\right.$ $\left.\left.C_{8} K_{n}\left(k_{p} r\right)\right]\right\} e^{i n \theta}$ 
where $Y_{n}\left(k_{0} r\right)$ and $K_{n}\left(k_{p} r\right)$ are the second kind Bessel function and second kind modified Bessel function of order $n$, where $k_{0}$ and $k_{p}$ satisfy the dispersion relations $\omega^{2}=g k_{0} \tanh \left(k_{0} d\right)=-g k_{p} \tan \left(k_{p} d\right)$ for $p=1,2, \ldots$.

The scattered potential must satisfy the Sommerfeld condition (22) in far field of the cylinder.

$$
\begin{aligned}
& \lim _{n \rightarrow \infty}\left\{\sqrt{r} \cosh \left(k_{0} z\right)\left[C_{5} \frac{d J_{n}\left(k_{0} r\right)}{d r}+C_{6} \frac{d Y_{n}\left(k_{0} r\right)}{d r}\right]\right. \\
& +\sum_{p=1}^{\infty} \sqrt{r} \cos \left(k_{p} z\right)\left[C_{7} \frac{d I_{n}\left(k_{p} r\right)}{d r}+C_{8} \frac{d K_{n}\left(k_{p} r\right)}{d r}\right] \\
& -i k_{0}\left(\sqrt{r} \cosh \left(k_{0} z\right)\left[C_{5} J_{n}\left(k_{0} r\right)+C_{6} Y_{n}\left(k_{0} r\right)\right]\right. \\
& \left.\left.+\sum_{p=1}^{\infty} \sqrt{r} \cos \left(k_{p} z\right)\left[C_{7} I_{n}\left(k_{p} r\right)+C_{8} K_{n}\left(k_{p} r\right)\right]\right)\right\} \\
& =0
\end{aligned}
$$

Substituting (26) into (22) and simplifying the expressions by using (A1) to (A6) in the Appendix, we have

$$
\begin{aligned}
& \sqrt{\frac{2}{\pi k_{0}}} \cosh \left(k_{0} z\right) \sin \left(k_{0} r-\frac{\pi}{4}-\frac{n \pi}{2}\right)\left(-C_{5}-i C_{6}\right)+ \\
& \sqrt{\frac{2}{\pi k_{0}}} \cosh \left(k_{0} z\right) \cos \left(k_{0} r-\frac{\pi}{4}-\frac{n \pi}{2}\right)\left(C_{6}-i C_{5}\right)=0 \\
& \text { If } \\
& \begin{array}{l}
r \rightarrow \infty \quad \rightarrow \quad I_{n}\left(k_{p} r\right) \rightarrow \infty \quad \rightarrow \quad C_{7}=0 \\
r \rightarrow \infty \quad \rightarrow \quad K_{n}\left(k_{p} r\right) \rightarrow 0
\end{array}
\end{aligned}
$$

By using (28) and considering (A7), the arbitrary constants are obtained as $C_{6}=i C_{5}$. Therefore, the scattered potential is generated as

$$
\begin{aligned}
& \varphi_{2 s}(r, \theta, z)= \\
& \frac{g H}{\omega} \sum_{n=-\infty}^{\infty}\left[C_{5} \cosh \left(k_{0} z\right) H_{n}^{(1)}\left(k_{0} r\right)+\sum_{p=1}^{\infty} C_{8} \cos \left(k_{p} z\right) K_{n}\left(k_{p} r\right)\right] e^{i n \theta}
\end{aligned}
$$

where $H_{n}^{(1)}\left(k_{0} r\right)$ is the first kind Hankel function of order $n$. The scattered potential in the exterior domain consists of two parts; one zone is the potential from the seabed to the bottom of the cylinder (region I) and the other is the 
potential from the bottom of the cylinder to the free surface (region II). For convenience, Eq.31 may be defined as follows:

$$
\begin{gathered}
\varphi_{2 s}(r, \theta, z)=\frac{g H}{\omega} \sum_{n=-\infty}^{\infty} \chi_{2 s}^{(n)}(r, z) e^{i n \theta} \\
\chi_{2 s}^{(n)}(r, z)=C_{9} \cosh \left(k_{0} z\right) H_{n}^{(1)}\left(k_{0} r\right)+ \\
C_{10} \cosh \left(k_{0} z\right) H_{n}^{(1)}\left(k_{0} r\right)+\sum_{p=1}^{\infty} C_{8} \cos \left(k_{p} z\right) K_{n}\left(k_{p} r\right)
\end{gathered}
$$

The no penetration condition (Eq.21) is introduced into the first part of equation (33) and based on Eq.34, one of the unknown coefficient is determined:

$$
C_{9} \cosh \left(k_{0} z\right) H_{n}^{(1)}\left(k_{0} r\right)=-i \frac{\cosh \left(k_{0} z\right)}{\cosh \left(k_{0} d\right)} J_{n}^{\prime}\left(k_{0} a\right) \rightarrow C_{9}=\frac{-i^{n}}{\cosh \left(k_{0} d\right)} \frac{J_{n}^{\prime}\left(k_{0} a\right)}{H_{n}^{(1)}{ }^{\prime}\left(k_{0} a\right)}
$$

The exterior potential that consists of incident and scattered wave fields ( $\varphi_{2}=$ $\left.\varphi_{2 I}+\varphi_{2 s}\right)$, is given by

$$
\begin{aligned}
\phi_{2}(r, \theta, z, t) & =\frac{g H}{\omega} \sum_{n=-\infty}^{\infty} \chi_{2}^{(n)}(r, z) e^{i n \theta} e^{-i \omega t} \\
\chi_{2}^{(n)}(r, z)= & i^{n} \frac{\cosh \left(k_{0} z\right)}{\cosh \left(k_{0} d\right)}\left[J_{n}\left(k_{0} r\right)-\frac{J_{n}^{\prime}\left(k_{0} a\right)}{H_{n}^{(1)}\left(k_{0} a\right)} H_{n}^{(1)}\left(k_{0} r\right)\right] \\
& +D_{n 0} \cdot H_{n}^{(1)}\left(k_{0} r\right) \cdot \cosh \left(k_{0} z\right)+\sum_{p=1}^{\infty} D_{n p} K_{n}\left(k_{p} r\right) \cos \left(k_{p} z\right)
\end{aligned}
$$

where the unknown coefficient $D_{n 0}$ can be determined by multiplying (37) by $\cosh \left(k_{0} z\right)$ and integrating with respect to $z$, from 0 to $d$ :

$$
\begin{aligned}
\frac{\partial \chi_{2}^{(n)}(a, z)}{\partial r}=D_{n 0} H_{n}^{(1)}{ }^{\prime}\left(k_{0} a\right) \cdot \cosh \left(k_{0} z\right)+\sum_{p=1}^{\infty} D_{n p} \cdot K_{n}{ }^{\prime}\left(k_{p} a\right) \cdot \cos \left(k_{p} z\right) \\
\begin{aligned}
\int_{0}^{d} \frac{\partial \chi_{2}^{(n)}(a, z)}{\partial r} \cosh \left(k_{0} z\right) d z & =D_{n 0} H_{n}^{(1)}{ }^{\prime}\left(k_{0} a\right) \int_{0}^{d} \cosh ^{2}\left(k_{0} z\right) d z \\
& +\sum_{p=1}^{\infty} D_{n p} K_{n}^{\prime}\left(k_{p} a\right) \int_{0}^{d} \cos \left(k_{p} z\right) \cdot \cosh \left(k_{0} z\right) d z
\end{aligned}
\end{aligned}
$$




$$
\begin{aligned}
\int_{0}^{d} \frac{\partial \chi_{2}^{(n)}(a, z)}{\partial r} \cosh \left(k_{0} z\right) d z & =d \cdot N_{0} \cdot H_{n}^{(1)^{\prime}}\left(k_{0} a\right) \cdot D_{n 0} \\
& +\sum_{p=1}^{\infty} D_{n p} \cdot K_{n}^{\prime}\left(k_{p} a\right)\left[\frac{k_{0} \cos \left(k_{p} d\right) \cdot \sinh \left(k_{0} d\right)+k_{p} \sin \left(k_{p} d\right) \cdot \cosh \left(k_{0} d\right)}{k_{0}{ }^{2}+k_{p}{ }^{2}}\right]
\end{aligned}
$$

Because of the relation $\omega^{2}=g k_{0} \tanh k_{0} d=-g k_{p} \tan k_{p} d$, the latest term on the right hand side of (39) is equal to zero. Moreover, $N_{0}$ and $N_{\mathrm{p}}$ can be defined as follows:

$N_{0}=\frac{1}{2}\left(1+\frac{\sinh \left(2 k_{0} d\right)}{2 k_{0} d}\right) \quad, \quad N_{p}=\frac{1}{2}\left(1+\frac{\sin \left(2 k_{p} d\right)}{2 k_{p} d}\right)$

Therefore, $D_{n 0}$ is found to be

$$
D_{n 0}=\frac{1}{d N_{0}^{\frac{1}{2}} H_{n}^{(1)}\left(k_{0} a\right)} \int_{0}^{d} \frac{\partial \chi_{2}^{(n)}}{\partial r} \frac{\cosh \left(k_{0} z\right)}{N_{0}^{\frac{1}{2}}} d z
$$

On the other hand, the unknown coefficient $D_{n p}$ can be determined by multiplying (37) by $\cos \left(k_{p} z\right), p=1,2, \ldots$ and integrating with respect to $z$, from 0 to $d$ :

$$
\begin{aligned}
\int_{0}^{d} \frac{\partial \chi_{2}^{(n)}(a, z)}{\partial r} \cos \left(k_{p} z\right) d z & =D_{n 0} H_{n}^{(1)}\left(k_{0} a\right) \int_{0}^{d} \cosh \left(k_{0} z\right) \cdot \cos \left(k_{p} z\right) d z \\
& +\sum_{p=1}^{\infty} D_{n p} K_{n}^{\prime}\left(k_{p} a\right) \int_{0}^{d} \cos ^{2}\left(k_{p} z\right) d z \\
\int_{0}^{d} \frac{\partial \chi_{2}^{(n)}(a, z)}{\partial r} \cos \left(k_{p} z\right) d z= & d \cdot N_{p} \cdot K_{n}^{\prime}\left(k_{p} a\right) \cdot D_{n p}
\end{aligned}
$$

Then, $D_{n p}$ is determined to be

$$
D_{n p}=\frac{1}{d N_{p}^{\frac{1}{2}} K_{n}^{\prime}\left(k_{p} a\right)} \int_{0}^{d} \frac{\partial \chi_{2}^{(n)}}{\partial r} \frac{\cos \left(k_{p} z\right)}{N_{p}^{\frac{1}{2}}} d z
$$

The new form of scattered potential is indicated below:

$$
\phi_{2}(r, \theta, z, t)=\frac{g H}{\omega} \sum_{n=-\infty}^{\infty} \chi_{2}^{(n)}(r, z) e^{i n \theta} e^{-i \omega t}
$$




$$
\begin{aligned}
\chi_{2}^{(n)}(r, z)= & i^{n} \frac{\cosh \left(k_{0} z\right)}{\cosh \left(k_{0} d\right)}\left[J_{n}\left(k_{0} r\right)-\frac{J_{n}^{\prime}\left(k_{0} a\right)}{H_{n}^{(1)}{ }^{\prime}\left(k_{0} a\right)} H_{n}^{(1)}\left(k_{0} r\right)\right] \\
& +D_{n 0} \frac{H_{n}^{(1)}\left(k_{0} r\right)}{H_{n}^{(1)}{ }^{\prime}\left(k_{0} a\right)} \frac{\cosh \left(k_{0} z\right)}{N_{0}^{\frac{1}{2}}}+\sum_{p=1}^{\infty} D_{n p} \frac{K_{n}\left(k_{p} r\right)}{K_{n}^{\prime}\left(k_{p} a\right)} \frac{\cos \left(k_{p} z\right)}{N_{p}^{\frac{1}{2}}}
\end{aligned}
$$

while, the new forms of coefficients are displayed as

$$
D_{n 0}=\frac{1}{k_{0} d} \int_{0}^{d} \frac{\partial \chi_{2}^{(n)}}{\partial r} \frac{\cosh \left(k_{0} z\right)}{N_{0}^{\frac{1}{2}}} d z \quad, \quad D_{n p}=\frac{1}{k_{p} d} \int_{0}^{d} \frac{\partial \chi_{2}^{(n)}}{\partial r} \frac{\cos \left(k_{p} z\right)}{N_{p}^{\frac{1}{2}}} d z
$$

\subsection{Determination of the Unknown Coefficients}

Matching conditions should be applied at the interface $r=a$ to determine the coefficients $C_{n m}$ and $D_{n p}$.

The first case that must be investigated is

$$
\chi_{n m}^{1}=\chi_{n p}^{2} \quad \text { on } \quad r=a, 0 \leq z \leq h
$$

Using (16) and (45) in (47), yields in

$$
\begin{aligned}
\frac{C_{n 0}}{2}+\sum_{m=1}^{\infty} C_{n m} \cos \left(\frac{m \pi z}{h}\right)= & i^{n} \frac{\cosh \left(k_{0} z\right)}{\cosh \left(k_{0} d\right)}\left[J_{n}\left(k_{0} r\right)-\frac{J_{n}^{\prime}\left(k_{0} a\right)}{H_{n}^{(1)}\left(k_{0} a\right)} H_{n}^{(1)}\left(k_{0} r\right)\right]+ \\
& D_{n 0} \frac{H_{n}^{(1)}\left(k_{0} r\right)}{H_{n}^{(1)}\left(k_{0} a\right)} \frac{\cosh \left(k_{0} z\right)}{N_{0}^{\frac{1}{2}}}+\sum_{p=1}^{\infty} D_{n p} \frac{K_{n}\left(k_{p} r\right)}{K_{n}\left(k_{p} a\right)} \frac{\cos \left(k_{p} z\right)}{N_{p}^{\frac{1}{2}}}
\end{aligned}
$$

By multiplying (48) by $\cos \left(\frac{m \pi z}{h}\right), m=0,1, \ldots$ and integrating with respect to $z$ over the region of validity, we get

$$
\begin{aligned}
& \frac{C_{n 0}}{2} \int_{0}^{h} \cos \left(\frac{m \pi z}{h}\right) d z+\sum_{m=1}^{\infty} C_{n m} \int_{0}^{h} \cos ^{2}\left(\frac{m \pi z}{h}\right) d z= \\
& i_{n}\left(k_{0} a\right)-J_{n}^{\prime}\left(k_{0} a\right) / H_{n}^{(1)}\left(k_{0} a\right) \\
& \cosh \left(k_{0} d\right) \\
& \frac{D_{n 0}^{(1)}\left(k_{0} a\right)}{N_{0}^{\frac{1}{2}}} \frac{H_{n}^{(1)}\left(k_{0} a\right)}{H_{n}^{(1)}\left(k_{0} a\right)} \int_{0}^{h} \cosh \left(k_{0} z\right) \cos \left(\frac{m \pi z}{h}\right) d z+\sum_{p=1}^{\infty} \frac{D_{n p}}{N_{p}^{\frac{1}{2}}} \frac{K_{n}\left(k_{0} z\right) \cdot \cos \left(\frac{m \pi}{h}\right) d z+}{K_{n}^{\prime}\left(k_{p} a\right)} \int_{0}^{h} \cos \left(k_{p} z\right) \cos \left(\frac{m \pi}{h}\right) d z
\end{aligned}
$$

To simplify (49), (A8) is used and at last one of the unknown coefficients is obtained as function of the other: 


$$
\begin{aligned}
C_{n m} & -\left[\frac{2 H_{n}^{(1)}\left(k_{0} a\right) \cdot k_{0} h^{2} \cdot(-1)^{m} \cdot \sinh \left(k_{0} h\right)}{h \cdot H_{n}^{(1)}\left(k_{0} a\right) \cdot N_{0}^{\frac{1}{2}} \cdot\left(k_{0}{ }^{2} h^{2}+m^{2} \pi^{2}\right)}\right] D_{n 0}-\sum_{p=1}^{\infty}\left[\frac{2 K_{n}\left(k_{p} a\right) \cdot k_{p} h^{2} \cdot(-1)^{m} \cdot \sin \left(k_{p} h\right)}{h \cdot K_{n}{ }^{\prime}\left(k_{p} a\right) \cdot N_{p}^{\frac{1}{2}} \cdot\left(k_{p}{ }^{2} h^{2}-m^{2} \pi^{2}\right)}\right] D_{n p}= \\
& \frac{2 i^{n} \cdot \sinh \left(k_{0} h\right) \cdot(-1)^{m} \cdot k_{0} h^{2}}{h \cosh \left(k_{0} d\right)\left(k_{0}{ }^{2} h^{2}+m^{2} \pi^{2}\right)} \frac{2 i}{\pi k_{0} a H_{n}^{(1)}{ }^{\prime}\left(k_{0} a\right)} \quad, \text { for } \quad m \geq 0
\end{aligned}
$$

The second case that must be satisfied is the gradient condition:

$$
\frac{\partial \chi_{n m}^{1}}{\partial r}=\frac{\partial \chi_{n p}^{2}}{\partial r} \quad, \quad r=a, 0 \leq z \leq h
$$

and by using the derivatives of $\chi_{n m}^{1}$ and $\chi_{n p}^{2}$ in (51), we have

$$
\frac{n C_{n 0}}{2 a}+\sum_{m=1}^{\infty} C_{n m} \frac{I_{n}^{\prime}\left(\frac{m \pi a}{h}\right)}{I_{n}\left(\frac{m \pi a}{h}\right)} \cos \left(\frac{m \pi z}{h}\right)=D_{n 0} \frac{\cosh \left(k_{0} z\right)}{N_{0}^{\frac{1}{2}}}+\sum_{p=1}^{\infty} D_{n p} \frac{\cos \left(k_{p} z\right)}{N_{p}^{\frac{1}{2}}}
$$

For $p=0$ and $m \geq 0,(52)$ can be written as follows:

$$
\frac{n C_{n 0}}{2 a} \int_{0}^{h} \cosh \left(k_{0} z\right) d z+\sum_{m=1}^{\infty} C_{n m} \frac{I_{n}^{\prime}\left(\frac{m \pi a}{h}\right)}{I_{n}\left(\frac{m \pi a}{h}\right)} \int_{0}^{h} \cos \left(\frac{m \pi a}{h}\right) \cdot \cosh \left(k_{0} z\right) d z=\frac{D_{n 0}}{N_{0}^{\frac{1}{2}}} \int_{0}^{h} \cosh ^{2}\left(k_{0} z\right) d z
$$

Therefore, $D_{n 0}$ is simplified to be

$$
D_{n 0}=\left[\frac{n \cdot \sinh \left(k_{0} h\right)}{2 a k_{0}{ }^{2} N_{0}^{\frac{1}{2}}}\right] C_{n 0}+\sum_{m=1}^{\infty}\left[\frac{I_{n}^{\prime}\left(\frac{m \pi a}{h}\right) h(-1)^{m} \sinh \left(k_{0} h\right)}{I_{n}\left(\frac{m \pi a}{h}\right)\left(k_{0}{ }^{2} h^{2}+m^{2} \pi^{2}\right) N_{0}^{\frac{1}{2}}}\right] C_{n m} \quad, \text { for } \quad p=0, m \geq 0
$$

Also, for $p>0$ and $m \geq 0$, (52) can be written as follows:

$$
\frac{n C_{n 0}}{2 a} \int_{0}^{h} \cos \left(k_{p} z\right) d z+\sum_{m=1}^{\infty} C_{n m} \frac{I_{n}^{\prime}\left(\frac{m \pi a}{h}\right)}{I_{n}\left(\frac{m \pi a}{h}\right)} \int_{0}^{h} \cos \left(\frac{m \pi a}{h}\right) \cdot \cos \left(k_{p} z\right) d z=\sum_{p=1}^{\infty} \frac{D_{n p}}{N_{p}^{\frac{1}{2}}} \int_{0}^{h} \cos ^{2}\left(k_{p} z\right) d z
$$

So, $D_{n 0}$ is obtained as in

$$
D_{n p}=\left[\frac{n \cdot \sin \left(k_{p} h\right)}{2 a k_{p}{ }^{2} h N_{p}^{\frac{1}{2}}}\right] C_{n 0}+\sum_{m=1}^{\infty}\left[\frac{I_{n}^{\prime}\left(\frac{m \pi a}{h}\right) h(-1)^{m} \sin \left(k_{p} h\right)}{I_{n}\left(\frac{m \pi a}{h}\right)\left(k_{p}{ }^{2} h^{2}-m^{2} \pi^{2}\right) N_{p}^{\frac{1}{2}}}\right] C_{n m} \quad, \text { for } \quad p>0, m \geq 0
$$

The unknown coefficients should be truncated after a finite number of terms and obtained numerically. 


\section{Discussion of Results}

\subsection{Heave force}

The heave excitation force is determined by integration of the fluid pressure over the lower region of the cylinder, while the dynamic pressure of the fluid is obtained from the linear Bernoulli equation. By substitution of relations (15) and (16) into the pressure equation, we get

$P=-\rho \frac{\partial \varphi_{1}}{\partial t}=-\rho(-i g H) \sum_{n=-\infty}^{\infty} \chi_{1}^{(n)}(r, h) e^{i n \theta} e^{-i \omega t}$

In continuation, by integration of the fluid pressure over the wet surface at the bottom of the cylinder, the heave excitation force is computed as

$$
F_{z}=\iint P d A=\iint P r d r d \theta
$$

Substitution of (57) into (58) would yield in

$$
\begin{aligned}
& F_{z}=i \rho g H \int_{0}^{2 \pi} \int_{0}^{a} \sum_{n=-\infty}^{\infty} \chi_{1}^{(n)}(r, h) e^{i n \theta} e^{-i \omega t}= \\
& i \rho g H \int_{0}^{a}\left[\sum_{n=-\infty}^{\infty} \chi_{1}^{(n)}(r, h) \int_{0}^{2 \pi} e^{i n \theta} d \theta\right] e^{-i \omega t} r d r \\
& \int_{0}^{2 \pi} e^{i n \theta} d \theta= \begin{cases}2 \pi & n=0 \\
0 & n \neq 0\end{cases}
\end{aligned}
$$

By using equation (60), it is observed that only the term $n=0$ for the given series would produce a nonzero answer for the force equation. Therefore, the heave excitation force can be obtained as follows:

$$
\begin{gathered}
F_{z}=i 2 \pi \rho g H \int_{0}^{a} \chi_{1}^{(0)}(r, h) e^{-i \omega t} r d r \\
=i 2 \pi \rho g H \int_{0}^{a}\left[\frac{C_{0}^{(0)}}{2}+\sum_{m=1}^{\infty} C_{m}^{(0)} \frac{I_{0}\left(\frac{m \pi r}{h}\right)}{I_{0}\left(\frac{m \pi a}{h}\right)}(-1)^{m}\right] e^{-i \omega t} r d r \\
=i 2 \pi \rho g H\left[\frac{C_{0}^{(0)}}{2} \int_{0}^{a} r d r+\sum_{m=1}^{\infty} \frac{C_{m}^{(0)}(-1)^{m}}{I_{0}\left(\frac{m \pi a}{h}\right)} \int_{0}^{a} r I_{0}\left(\frac{m \pi r}{h}\right) d r\right] e^{-i \omega t}
\end{gathered}
$$

Further simplification of this, along with using (A9), would result in

$F_{z}=i 2 \pi \rho g H\left[\frac{C_{0}^{(0)} a^{2}}{4}+\sum_{m=1}^{\infty} C_{m}^{(0)} \frac{I_{1}\left(\frac{m \pi a}{h}\right)}{I_{0}\left(\frac{m \pi a}{h}\right)} \frac{h a(-1)^{m}}{m \pi}\right] e^{-i \omega t}$ 


\subsection{Surge force}

Surge excitation force is also calculated by integration of the fluid pressure around the cylinder in the x-direction. Substitution of equations (35) and (36) into the integration formula of fluid pressure, would lead to

$$
\begin{aligned}
& F_{x}=-\iint-\rho \frac{\partial \emptyset_{2}}{\partial t} \cos \theta d A \\
& =\rho(-i \omega) \iint \frac{g H}{\omega} \sum_{n=-\infty}^{\infty} \chi_{2}^{(n)}(a, z) e^{i n \theta} e^{-i \omega t} \cos \theta d A \\
& =-i \rho g H \int_{h}^{d} \int_{0}^{2 \pi} \sum_{n=-\infty}^{\infty}\left\{i^{n} \frac{\cosh \left(k_{0} z\right)}{\cosh \left(k_{0} d\right)}\left[J_{n}\left(k_{0} a\right)-\frac{J_{n}^{\prime}\left(k_{0} a\right)}{H_{n}^{(1)}{ }^{\prime}\left(k_{0} a\right)} H_{n}^{(1)}\left(k_{0} a\right)\right]\right. \\
& +D_{n 0} \frac{H_{n}\left(k_{0} a\right)}{H_{n}^{(1)}\left(k_{0} a\right)} \frac{\cosh \left(k_{0} z\right)}{N_{0}^{1 / 2}} \\
& \left.+\sum_{p=1}^{\infty} D_{n p} \frac{K_{n}\left(k_{p} a\right)}{K_{n}^{\prime}\left(k_{p} a\right)} \frac{\cos \left(k_{p} z\right)}{N_{p}^{1 / 2}}\right\} e^{i n \theta} e^{-i \omega t} \cos \theta a d \theta d z
\end{aligned}
$$

After simplification of equation (63) and using relation (A8) and equation (64), it is determined that the surge excitation force only becomes nonzero, if $n= \pm 1$ :

$$
\begin{gathered}
\left\{\begin{array}{l}
n= \pm 1 \rightarrow \int_{0}^{2 \pi} e^{i n \theta} \cos \theta \quad d \theta=\pi \\
n \neq \pm 1 \rightarrow \int_{0}^{2 \pi} e^{i n \theta} \cos \theta \quad d \theta=0
\end{array}\right. \\
F_{x}=-i \rho \pi g a H \int_{h}^{d}\left\{\frac{2 i \cosh \left(k_{0} z\right)}{\cosh \left(k_{0} d\right)}\left[\frac{2 i}{\pi k_{0} a H_{n}^{(1)}{ }^{\prime}\left(k_{0} a\right)}\right]+\sum_{p=0}^{\infty}\left[D_{(-1) p}+D_{(1) p}\right] \frac{V_{1}\left(k_{p} a\right)}{V_{1}^{\prime}\left(k_{p} a\right)} Z_{p}(z)\right\} e^{-i \omega t} d z
\end{gathered}
$$

By integrating (65) and using (A10), the horizontal force acting on the cylinder is found to be 


$$
\begin{aligned}
F_{x}= & -i \rho \pi g a H \frac{2 i\left[\sinh \left(k_{0} d\right)-\sinh \left(k_{0} h\right)\right]}{k_{0} \cosh \left(k_{0} d\right)}\left[\frac{2 i}{\pi k_{0} a H_{n}^{(1)}{ }^{\prime}\left(k_{0} a\right)}\right] \\
& +\sum_{p=0}^{\infty}\left[D_{(-1) p}+D_{(1) p}\right] \frac{V_{1}\left(k_{p} a\right)}{V_{1}^{\prime}\left(k_{p} a\right)} \frac{\gamma(p)}{k_{p} N_{p}^{\frac{1}{2}}} e^{-i \omega t} d z
\end{aligned}
$$

Where

$$
\left\{\begin{array}{cll}
V_{n}\left(k_{0} r\right)=H_{n}^{(1)}\left(k_{0} r\right), & \gamma(0)=\sinh \left(k_{0} d\right)-\sinh \left(k_{0} h\right), & p=0 \\
V_{n}\left(k_{p} r\right)=K_{n}\left(k_{p} r\right), & \gamma(p)=\sin \left(k_{0} d\right)-\sin \left(k_{0} h\right), & p>0
\end{array}\right.
$$

\subsection{Pitch Moment}

The pitch excitation moment around the cylinder with respect to the center of cylindrical coordinates $(\mathrm{O})$ is also found by integrating the fluid pressure in the direction of $\mathrm{x}$. By substituting the equations (35) and (36) in the fluid pressure integral around the cylinder, we get

$M_{x}=-\iint(P \cos \theta)(d-z) d A=-\iint-\rho \frac{\partial \varphi_{2}}{\partial t}(d-z) a d \theta d z$

Similar as in the case of the horizontal force, the pitch moment is also nonzero only for $n= \pm 1$ :

$$
M_{x}=\rho(-i g H) a \int_{0}^{2 \pi} \int_{h}^{d} \sum_{n=-\infty}^{\infty} \chi_{2}^{(n)}(a, z)(d-z) \cos \theta e^{i n \theta} e^{-i \omega t} d \theta d z
$$

Using (A11), equation (69) is simplified and written as follows:

$$
\begin{aligned}
& M_{x}=-i \pi \rho g a H \int_{h}^{d}\left[\chi_{(1)}^{2}(a, z)(d-z) d z+\chi_{(-1)}^{2}(a, z)(d-z) d z\right] e^{-i \omega t} \\
& =-i \pi \rho g a H \int_{h}^{d}\left\{\frac{2 i \cosh \left(k_{0} z\right)}{\cosh \left(k_{0} d\right)}\left[\frac{2 i}{\pi k_{0} a H_{n}^{(1)}\left(k_{0} a\right)}\right]+\sum_{p=0}^{\infty}\left[D_{(-1) p}+\right.\right. \\
& \left.\left.D_{(1) p}\right] \frac{V_{1}\left(k_{p} a\right)}{V_{1}^{\prime}\left(k_{p} a\right)} Z_{p}(z)\right\}(d-z) e^{-i \omega t} d z
\end{aligned}
$$

Ultimately, by using (A8) and reorganizing, the final form of the pitch moment about point $\mathrm{O}$ is written as 
$M_{x}=$

$-i \pi \rho g a H\left\{\frac{2 i}{\cosh \left(k_{0} d\right)}\left[\frac{2 i \xi(0)}{\pi k_{0} a H_{n}^{(1)}{ }^{\prime}\left(k_{0} a\right)}\right]+\right.$

$\left.\sum_{p=0}^{\infty}\left[D_{(-1) p}+D_{(1) p}\right] \frac{V_{1}\left(k_{p} a\right)}{V_{1}^{\prime}\left(k_{p} a\right)} \frac{\xi(p)}{N_{p}^{1 / 2}}\right\} e^{-i \omega t}$

where

$\left\{\begin{array}{l}\xi(0)=\int_{h}^{d} \cosh \left(k_{0} z\right)(d-z) d z=\frac{(h-d) \sinh \left(k_{h} h\right)}{k_{0}}+\frac{\cosh \left(k_{0} d\right)-\cosh \left(k_{0} h\right)}{k_{0}{ }^{2}} \\ \xi(p)=\int_{h}^{d} \cos \left(k_{p} z\right)(d-z) d z=\frac{(h-d) \sin \left(k_{p} h\right)}{k_{p}}+\frac{\cos \left(k_{p} h\right)-\cos \left(k_{p} h\right)}{k_{p}{ }^{2}}\end{array}\right.$

\section{Added Mass and Damping Coefficient}

For determination of the dynamic response of a floating cylinder, its dynamic equations must be solved. However, since the cylinder is vertical and symmetric, its degrees of freedom reduce to surge, heave, and pitch motions. The dynamic equation of a floating object used for calculating its displacement is as follows

$M \ddot{X}=F_{d}+F_{h y}+F_{R}$

where

$F_{d}$ : The excitation force resulting from solution of diffraction problem around the cylinder which is found by integration of pressure distribution over its surface.

$F_{h y}$ : The hydrostatic restoring force (linearized) depending on the displacement, which is expressed as

$F_{h y}=-K X$

where $K$ is the stiffness matrix and $X$ is the displacement vector of the floating body. Since the floating body is not moored, its stiffness matrix only includes the hydrostatic components.

$F_{R}$ : The resulting force from solution of radiation problem which is divided into components of force in acceleration and velocity phases. The weight force and the damping force are proportional to the acceleration and velocity vectors of the floating body, respectively.

$F_{R}=-m \ddot{X}-C \dot{X}$ 
where $m$ is the added mass matrix, $C$ is the damping matrix, and $\dot{X}$ and $\ddot{X}$ are the velocity and acceleration vectors of the floating body, respectively. Therefore, by substitution of equations (74) and (75) into (73), the dynamic equation is found to be

$(M+m) \ddot{X}+C \dot{X}+K X=F_{d}$.

For establishing the dynamic equation, it is necessary to determine the added mass, damping, and stiffness matrices of the vertical cylinder. Obviously, since the floating cylinder is not moored and controlling the surge motion is only possible through having the mooring system, the dynamic equation can only be established for the heave and pitch motions.

In this article, in order to calculate the added mass and damping coefficients of the pitch motion of the body, the strip theory is applied.

\subsection{Calculation of the Added Mass of the Floating System}

Hydrodynamic coefficients of the heave motion cannot be determined by the strip theory and should be found from the submerged disk method [19, 20, 21]. In submerged disk method, the bottom of the cylinder must be placed much farther from the fluid free surface and the heave motion of the floating cylinder is analogized to a two dimensional disk. This is done in a way that the cylinder cuts the water surface and that only one of its sides accelerates and its added mass is halved as in

$m_{33}=\frac{1}{2}\left(\frac{8}{3} \rho r^{3}\right)$

where $m_{33}$ is the added mass of the cylinder in heave motion, $\rho$ is the fluid density, and $r$ is the cylinder radius.

Since the floating cylinder is vertical and symmetric, we have

$m_{35}=m_{53}=0$.

Ultimately, the added mass for pitch motion, by using strip theory [19], becomes

$m_{55}=C_{m} \rho \pi r^{2}\left(\frac{(d-h)^{3}}{3}+\overline{K G}^{2}(d-h)+\overline{K G}(d-h)^{2}\right)$

where $m_{55}$ is the added mass of the pitch motion of the floating cylinder, $C_{m}$ is the added mass the added mass coefficient of the surge motion of the cylinder, $d$ is the water depth, $h$ is the distance from the seabed to the bottom of the cylinder, and $\overline{K G}$ is the distance from the center of gravity of the cylinder to its bottom. 


\subsection{Calculation of the floating cylinder damping}

The damping coefficient is also found similar to the method of finding the added mass [19]. Accordingly, the damping coefficient of the cylinder is first determined for a two-dimensional strip and subsequently for a three dimensional body.

$c_{33}=0$

$c_{35}=c_{53}=0$

$c_{55}=$
$\begin{cases}c_{11}\left(\frac{(d-h)^{3}}{4}+\overline{K G}(d-h)^{2}+\frac{3}{2} \overline{K G}^{2}(d-h)+\overline{K G}^{3}\right) & \text { for } \overline{K G}<h \\ c_{11}\left(\frac{\overline{K G}^{4}}{2(d-h)}+\frac{(d-h)^{3}}{4}+\overline{K G}(d-h)^{2}+\frac{3}{2} \overline{K G}^{2}(d-h)+\overline{K G}^{3}\right) & \text { for } \quad \overline{K G} \geq h\end{cases}$

where $c_{i j}$ are the damping coefficients of the floating cylinder. On the other hand, $c_{11}$ which is the damping coefficient of the surge motion of the cylinder, is calculated as in

$c_{11}=C_{D} \rho r(d-h)$

where the drag force coefficient for the cylinder is equal to $C_{D}=0.6$ [19].

\subsection{Calculation of the stiffness of the floating cylinder}

Since the floating cylinder is not moored, the restoring force is only due to the hydrostatic components of the cylinder which is only nonzero for the two following components:

$k_{33}^{h y}=\rho g A_{w p}=\rho g \pi a^{2}$

$k_{55}^{h y}=\rho g \forall \overline{\mathrm{GM}}_{1}=\rho g \forall(\overline{K G}-\overline{K B})+\rho g \frac{\pi a^{4}}{4}$

where $\forall$ is the submerged volume of the cylinder, and $\overline{K B}$ is the distance from the bottom to the center of buoyancy of the cylinder.

By determining the values of the added mass and damping of the body, equation (76) can be solved and eventually the dynamic response for the two motions of heave and pitch of the floating cylinder may be found. 


\section{Numerical Results}

Parametric studies of the effects of the wave excitation force on the floating cylinder have been performed for two different cases and plots of the wave excitation forces and moments have been sketched in terms of $a k_{0}$.

\section{Case 1:}

Variations of the water depth and the distance of the cylinder bottom from the seabed are studied, assuming that the draft of the cylinder is kept constant. The aim of this study is to investigate the influence of water depth on the forces exerted by the wave on the floating cylinder. Accordingly, the excitation forces and moments are calculated for three different water depths. Assuming that $B=\rho g \pi a(d-h) H$, the heave and surge forces as well as the pitch moment for this case are plotted in Figs.2, 3, and 4.

\section{Case 2:}

In this section, the water depth is kept constant and the effect of change in the draft of the floating cylinder on the forces exerted by the wave is studied. Accordingly, the excitation forces and moment of the wave are calculated and analyzed for three different distances from the seabed to the bottom of the cylinder. Once again, by assuming $=\rho g \pi a(d-h) H$, the plots of heave and surge forces as well as the pitch moment are presented in Figs.5, 6, and 7.

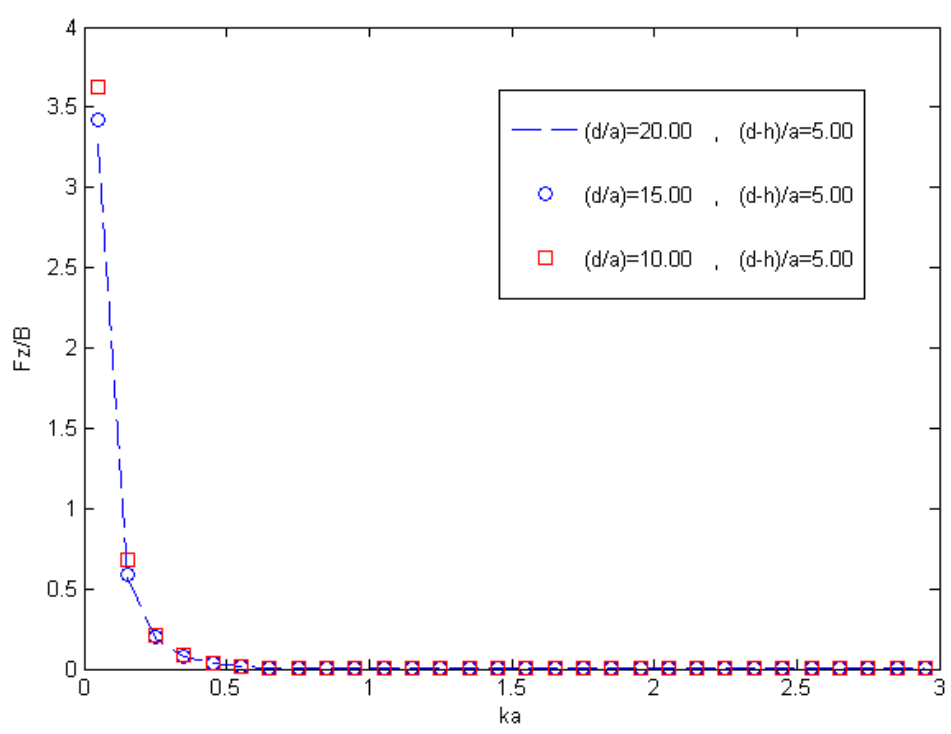

Fig.2. Non-dimensional heave force - Effect of different water depths at constant draft of the cylinder. 


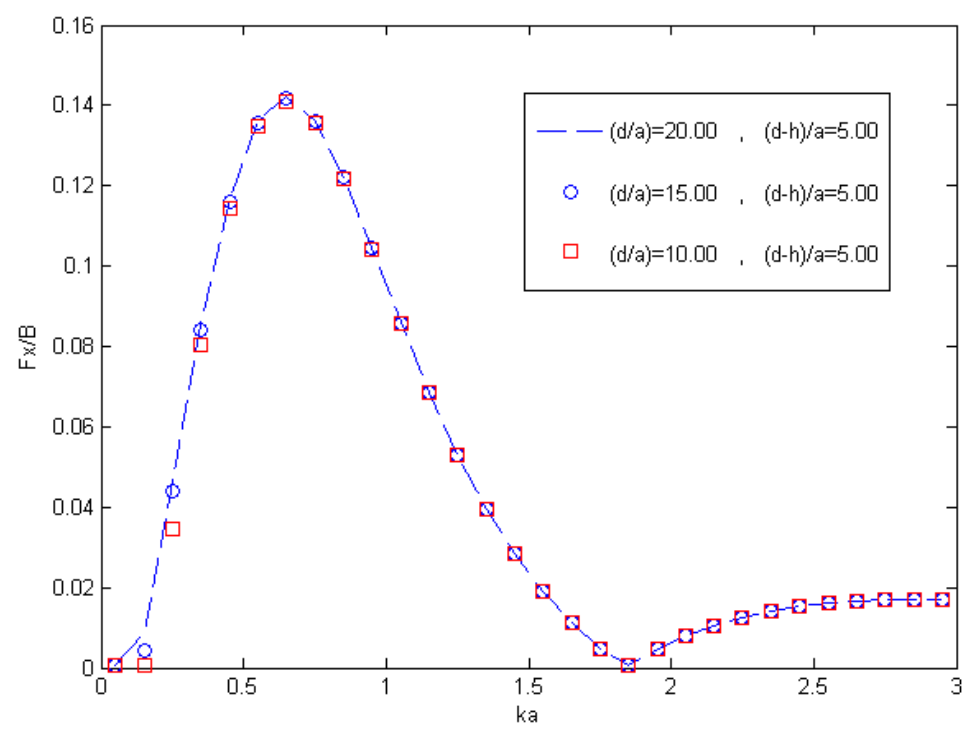

Fig.3. Non-dimensional surge force - Effect of different water depths at constant draft of the cylinder.

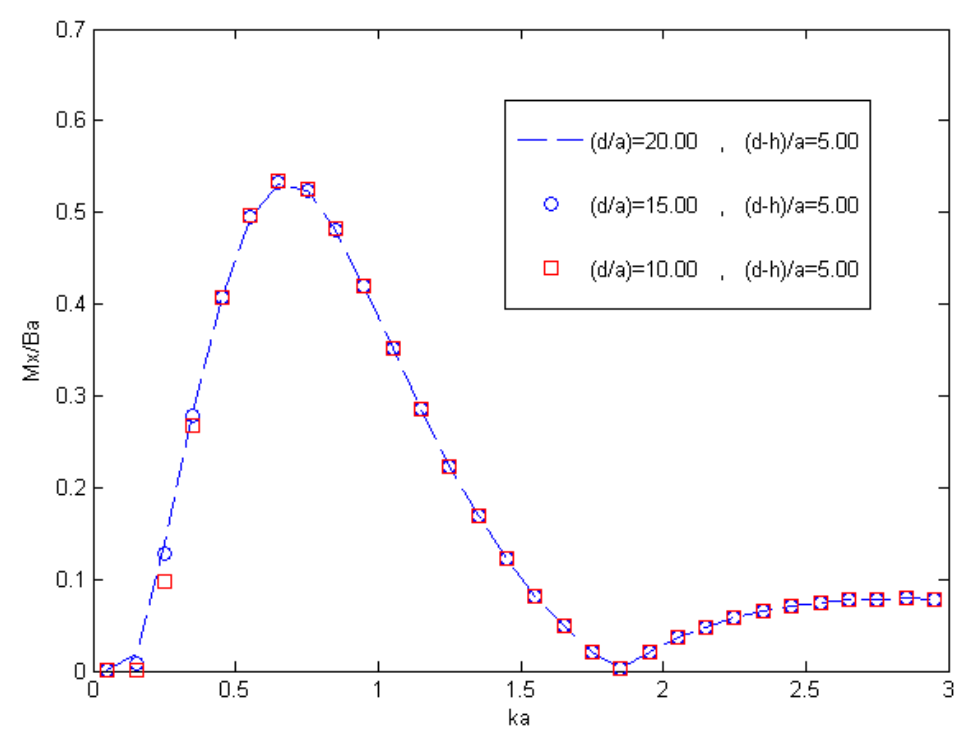

Fig.4. Non-dimensional pitch moment - Effect of different water depths at constant draft of the cylinder. 


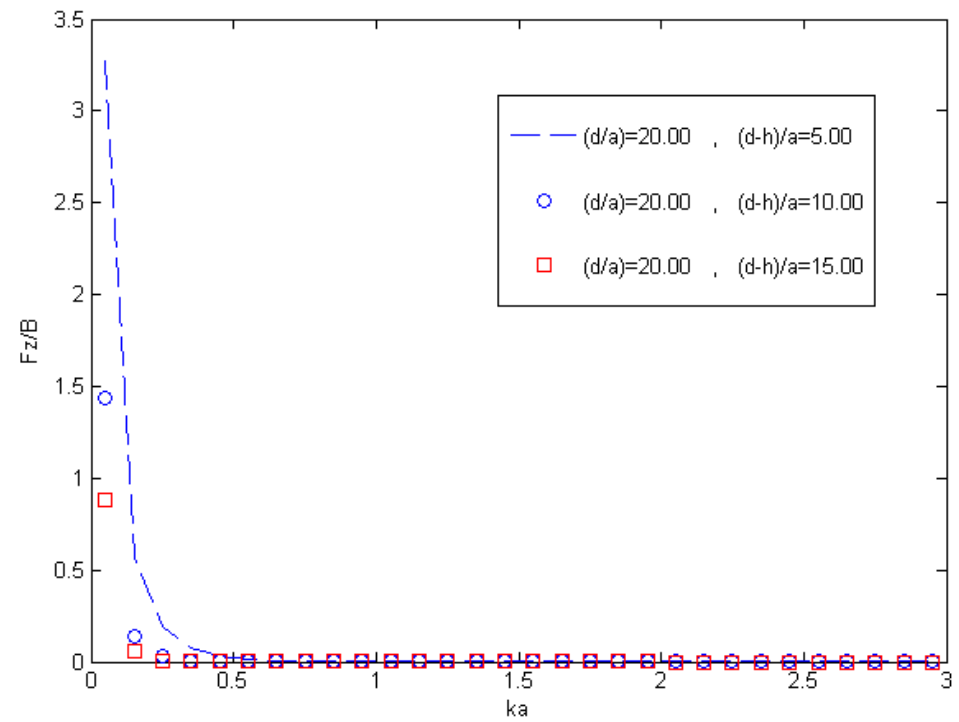

Fig.5. Non-dimensional heave force - Effect of different drafts of the cylinder at constant water depth.

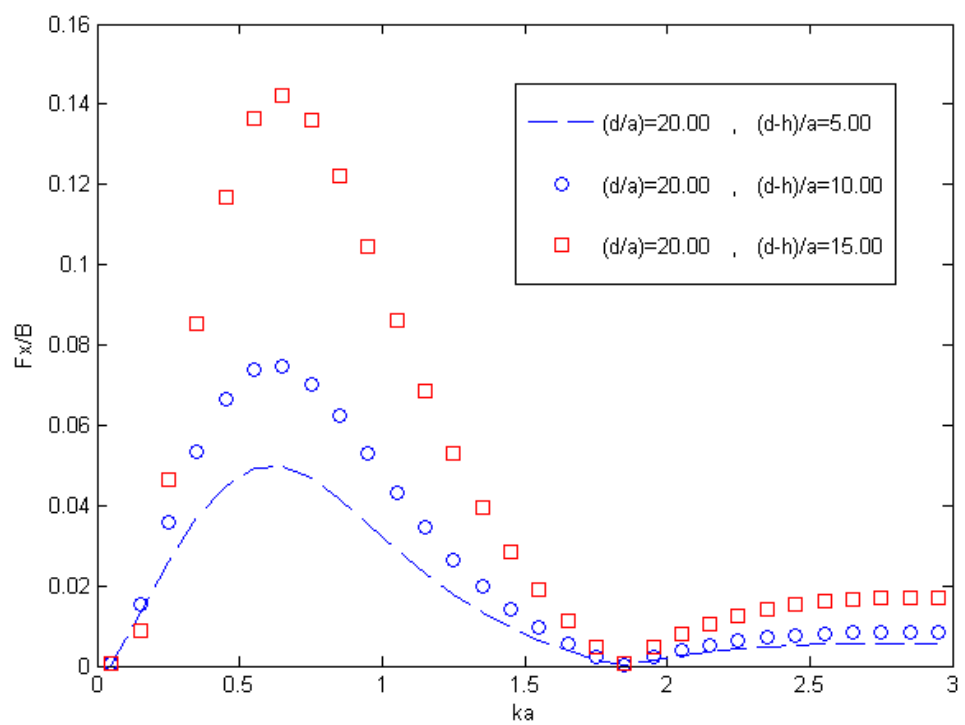

Fig.6. Non-dimensional surge force - Effect of different drafts of the cylinder at constant water depth. 


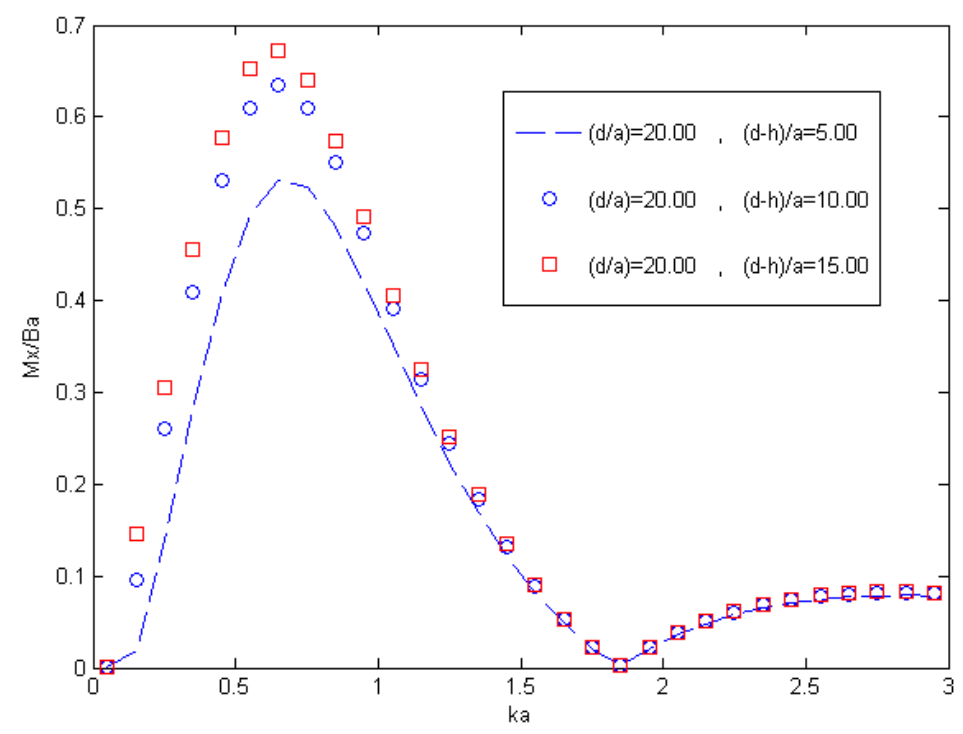

Fig.7. Non-dimensional pitch moment - Effect of different drafts of the cylinder at constantwater depth.

\section{Conclusion}

In this article, effort has been made to use the analytical solution of the diffraction problem for a cylinder of particular radius $(a)$ floating in a channel of specific depth $(d)$ and investigate the effects of geometrical parameters on the dynamics of this cylinder. To accomplish this task, Laplace's equation has been analytically solved under certain boundary conditions for the cylinder, which has gone under the water by the amount of $(d-h)$, with $h$ being the distance from the bottom of the channel to the bottom of the cylinder.

In these computations, a regular wave with the amplitude $\mathrm{H}$ intersects the floating cylinder and results in diffraction phenomenon. After determining the diffraction potential, the heave and surge, forces along with pitch moments for the cylinder were calculated. Having found the forces, the dynamic equation of the floating cylinder has been solved. In this article, two important dynamic motions (i.e. heave and pitch) of the cylinder have been analyzed. Since there is no mooring system considered in the surge motion, the dynamic investigation of this motion is ignored.

In order to study the effects of different parameters on the dynamics of the cylinder, two parameters of water depth and draft of the cylinder have been considered, albeit the values of these parameters were non-dimensionalized with respect to the cylinder radius. Based on the studies performed in this paper, the obtained results can be summarized as follows: 
1. By considering the draft as constant and increasing the water depth, the pitch moment as well as the heave and surge forces do not change significantly.

2. By keeping the water depth constant and increasing the draft, the heave force reduces while the pitch moment and the surge force increase.

3. Based on the obtained plots, it is observed that the heave and pitch motions of the cylinder do not vary much at the constant draft. As such, it can be stated that the effects of changes of water depth on the dynamics of the cylinder diminish, when the draft is considered constant.

4. As the cylinder descends more in the water, the range of heave and pitch motions reduce with an increase in the wave frequency. Based on the plots generated, the range of pitch angle at constant draft and low frequency is approximately $6 \%$ of the wave slope.

\section{References}

[1] Zheng, Y. H., Y. M. Shen, Y. G. You, B. J. Wu, and L. Rong,"Hydrodynamic properties of two vertical truncated cylinders in waves", Ocean Engineering, Vol.32, (2005), pp. 241-271.

[2] Miles, J.W. and J.F. Gilbert, "Scattering of gravity waves by a circular dock", Journal of Fluid Mechanics, Vol.34, (1968), pp. 783-793.

[3] Garret, C.J.R., " Wave forces on a circular dock", Journal of Fluid Mechanics, Cambridge, U.K., Vol.46, (1971), pp. 129-139.

[4] Black, J.L., C.C. Mei, and M.C.G. Bray, "Radiation and scattering of water waves by rigid bodies", Journal of Fluid Mechanics, Vol.46, (1971), pp. $151-164$.

[5] Isaacson, M.,'Wave forces on compound cylinders", Proc. Civil Engineering in the Oceans IV, I, ASCE, San Francisco, (1979), pp. 518-530.

[6] Yeung, R.W., "Added mass and damping of a vertical cylinder in finitedepth waters", Applied Ocean Research, Vol.3, (1981), pp. 119-133.

[7] Sabuncu, T. and S. Calisal, "Hydrodynamic coefficients for vertical circular cylinders at finite depth", Ocean Engineering, Vol.8, (1981), pp. 25-63.

[8] Calisal, S.M. and T. Sabuncu, "Hydrodynamic coefficients for vertical composite cylinders", Ocean Engineering, Vol.11, (1984), pp. 529-542.

[9] Calisal, S.M. and T. Sabuncu, "A generalized method for the calculation of hydrodynamic forces on discontinuous vertical cylinders", Ocean Engineering, Vol.20, (1993), pp. 1-18.

[10] Yeung, R.W. and S.H. Sphaier, "Wave-interference effects on a truncated cylinder in a channel", Journal of Engineering Mathematics, Vol.23, (1989), pp. 95-117. 
[11] Berggren, L. and M. Johansson, "Hydrodynamic coefficients of a wave energy device consisting of a buoy and a submerged plate", Applied Ocean Research, Vol.14, (1992), pp. 51-58.

[12] Eidsmoen, H., "Hydrodynamic parameters for a two-body axisymmetric system”, Applied Ocean Research, Vol.17, (1995), pp. 103-115.

[13] Bhatta, D.D. and M. Rahman, "Wave loadings on a vertical cylinder due to heave motion", International Journal of Mathematics and Mathematical Sciences, Vol.18, (1995), pp. 151-170.

[14] Bhatta, D.D. and M. Rahman, "On scattering and radiation problem for a cylinder in water of finite depth", International Journal of Engineering Science, Vol.41, (2003), pp. 931-967.

[15] Williams, A.N. W. Li, and K.H. Wang, "Water wave interaction with a floating porous cylinder", Ocean Engineering, Vol.27, (2000), pp. 1-28.

[16] Wu, B.J., Y.H. Zheng,Y.G. You, X.Y. Sun, and Y. Chen, "On diffraction and radiation problem for a cylinder over a caisson in water of finite depth", International Journal of Engineering Science, Vol.42, (2004), pp.1193-1213.

[17] Huang, J.B. and R. Eatock Taylor, "Semi-analytical solution for secondorder wave diffraction by a truncated circular cylinder in monochromatic waves", Journal of Fluid Mechanics, Vol.319, (1996), pp. 171-196.

[18] Mavrakos, S.A. and I.K. Chatjigeorgiou, "Second-order hydrodynamic effects on an arrangement of two concentric truncated vertical cylinders", Marine Structures, Vol.22, (2009), pp. 545-575.

[19] Mansouri, R. and H. Hadidi, "Comprehensive Study on the Linear Hydrodynamic Analysis of a Truss Spar in Random Waves", World Academy of Science, Engineering and Technology, Vol.53, (2009), pp. 930942.

[20] Patel, M.H., "Dynamics of Offshore Structures", Butterworth-Heinemann, (1989), ISBN 0-408- 01074-6.

[21] Newman, J. N., "Marine Hydrodynamics", (1977), MIT Press, Cambridge,UK. 